\title{
Crystal field excitations in electron superconductors
}

\author{
A T BOOTHROYD, S M DOYLE, D McK PAUL, D S MISRA \\ and $\mathrm{R}$ OSBORN* \\ Department of Physics, University of Warwick, Coventry, CV4 7AL, U.K. \\ *Rutherford Appleton Laboratory, Chilton, Didcot, Oxon, OX110QX, U.K.
}

\begin{abstract}
We have measured the complete crystalline electric field (CEF) spectrum of $\mathrm{Nd}^{3+}$ in superconducting $\mathrm{Nd}_{1.85} \mathrm{Ce}_{0.15} \mathrm{CuO}_{4}$ and $\mathrm{Nd}_{2} \mathrm{CuO}_{3.7} \mathrm{~F}_{0 \cdot 3}$, and in the non-superconducting parent compound, $\mathrm{Nd}_{2} \mathrm{CuO}_{4}$, by neutron inelastic scattering. The best description of the ground-state excitations for both compounds is achieved by the addition of a molecular field parameter to the CEF hamiltonian that takes account of exchange interactions from $\mathrm{Nd}$ or $\mathrm{Cu}$ spin ordering.
\end{abstract}

Keywords. Neutron scattering (inelastic); crystal field; magnetic interactions; $\mathrm{Nd}_{2-x} \mathrm{Ce}_{x} \mathrm{CuO}_{4}$.

\section{Introduction}

Earlier this year it was announced (Tokura et al 1989) that a new family of hightemperature superconductors had been discovered with the $\mathrm{Nd}_{2} \mathrm{CuO}_{4}$ structure where $\mathrm{Nd}$ can be replaced with $\mathrm{Pr}$ or $\mathrm{Sm}$. When these compounds are doped with $\mathrm{Ce}$ on the rare earth site or $\mathrm{F}$ on the oxygen site they provide the first examples of high-temperature superconductors with $n$-type rather than $p$-type conductivity.

Structurally, $\mathrm{Nd}_{2} \mathrm{CuO}_{4}$ differs from $\mathrm{La}_{2} \mathrm{CuO}_{4}$, a parent compound of some of the hole-doped superconductors, in the arrangement of oxygen around the copper ion. In the latter material the copper is octahedrally coordinated, whereas it is in a squareplanar environment in $\mathrm{Nd}_{2} \mathrm{CuO}_{4}$. In contrast to the $\mathrm{Sr}$-doped $\mathrm{La}_{2} \mathrm{CuO}_{4}$ superconductors, the new electron-doped materials have magnetic rare-earth ions situated above and below the $\mathrm{CuO}$ planes, and this provides us with an excellent opportunity to compare, by means of the crystalline electric field (CEF) excitations, the electronic and magnetic interactions at the rare-earth site in the superconductor and non-superconductor.

In this paper we report on measurements and analysis of the CEF spectra for superconducting $\mathrm{Nd}_{1.85} \mathrm{Ce}_{0.15} \mathrm{CuO}_{4}$ and $\mathrm{Nd}_{2} \mathrm{CuO}_{3.7} \mathrm{~F}_{0.3}$ and for their parent compound, $\mathrm{Nd}_{2} \mathrm{CuO}_{4}$. Earlier predictions for the crystal field levels from susceptibility data (Saez-Puche et al 1983; Seaman et al 1989) assumed a cubic environment for the rare-earth ion, and found a first-excited state either 40 or $70 \mathrm{meV}$ above the ground state, and a second state $100 \mathrm{meV}$ higher. Our measurements reveal a very different crystal-field scheme, whose description requires the full tetragonal symmetry of the rare-earth site, and which is modified by magnetic interactions.

\section{Experimental}

The polycrystalline samples used for these experiments were prepared by solid-state reaction. Appropriate quantities of $\mathrm{Nd}_{2} \mathrm{O}_{3}, \mathrm{CuO}, \mathrm{CeO}_{2}$ and $\mathrm{NdF}_{3}$ were mixed together and then reacted, with intermittent regrindings. The powders were pressed into 
cylindrical pellets of diameter $16 \mathrm{~mm}$ and thickness $3 \mathrm{~mm}$, sintered, and then annealed. Processing temperatures for the undoped and Ce-doped compounds are published elsewhere (Boothroyd et al 1989); the F-doped material was reacted and sintered at $900^{\circ} \mathrm{C}$ before annealing in nitrogen at the same temperature. $X$-ray powder diffraction patterns showed that the products were single phase, and superconductivity was observed in the doped samples by a.c. susceptibility.

For the neutron scattering measurements the sample was constructed of 20 to 30 pellets, stacked in an approximately square array with the cylinder axes perpendicular to the incident neutron beam. The pellets were separated by cadmium strips to prevent neutrons scattered in one pellet from travelling into adjacent pellets and being rescattered. The neutron measurements were made on the high energy transfer (HET) spectrometer at the ISIS pulsed neutron source of the Rutherford-Appleton Laboratory.

\section{Analysis and results}

In elementary crystal field theory it is usual to assume that the admixture of states within different $J$-levels can be neglected. With the crystallographic $c$-axis as the quantization direction, the crystal field hamiltonian for the tetragonal symmetry of the rare-earth site in $\mathrm{Nd}_{2-x} \mathrm{Ce}_{x} \mathrm{CuO}_{4}$ is then given by

$$
H_{\mathrm{CEF}}=B_{2}^{0} O_{2}^{0}+B_{4}^{0} O_{4}^{0}+B_{6}^{0} O_{6}^{0}+B_{4}^{4} O_{4}^{4}+B_{6}^{4} O_{6}^{4} \text {, }
$$

where the $B_{2 n}^{2 m}$ are the CEF parameters, and the $O_{2 n}^{2 m}$ are the corresponding Stevens' operators (Furrer et al 1988). The Hund's Rule ground state of $\mathrm{Nd}^{3+}$ has $J=\frac{9}{2}$ and, in general, the Hamiltonian (1) causes a splitting of the ten-fold degenerate $J$-multiplet into five doublets.

The neutron spectra measured at the lowest temperature with incident energies of $40 \mathrm{meV}$, are shown for $\mathrm{Nd}_{2} \mathrm{CuO}_{4}$ in figure 1 , for $\mathrm{Nd}_{1.85} \mathrm{Ce}_{0.15} \mathrm{CuO}_{4}$ in figure 2 and for $\mathrm{Nd}_{2} \mathrm{CuO}_{3 \cdot 7} \mathrm{~F}_{0 \cdot 3}$ in figure 3 . All three compounds have well-defined peaks at about

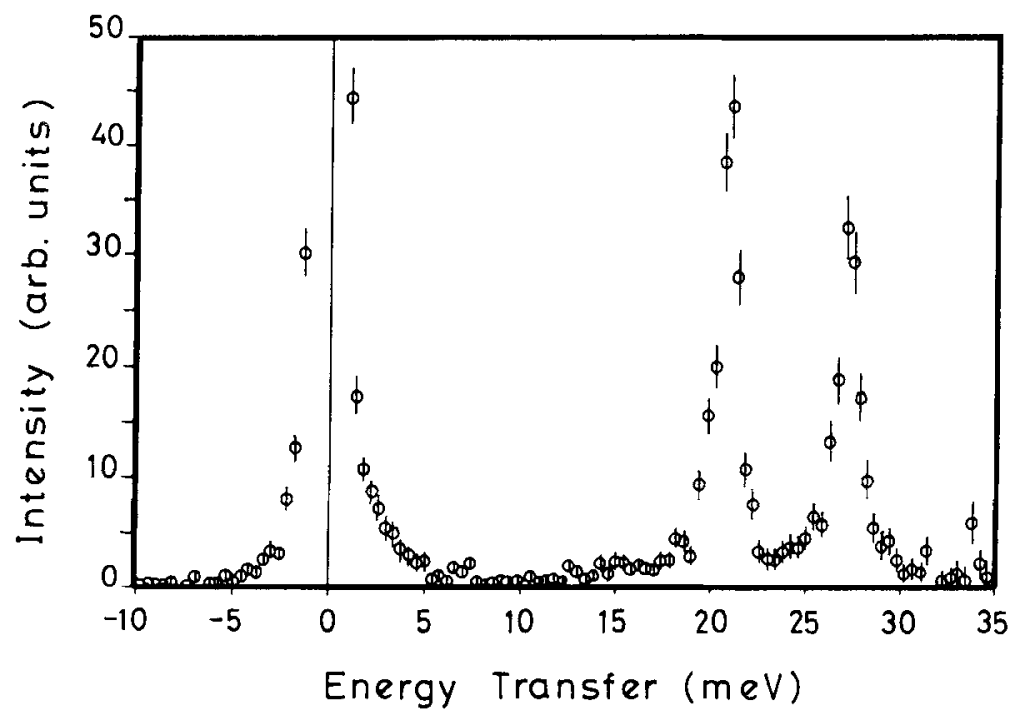

Figure 1. Crystal field transitions of $\mathrm{Nd}_{2} \mathrm{CuO}_{4}$ at $1.6 \mathrm{~K}$. 


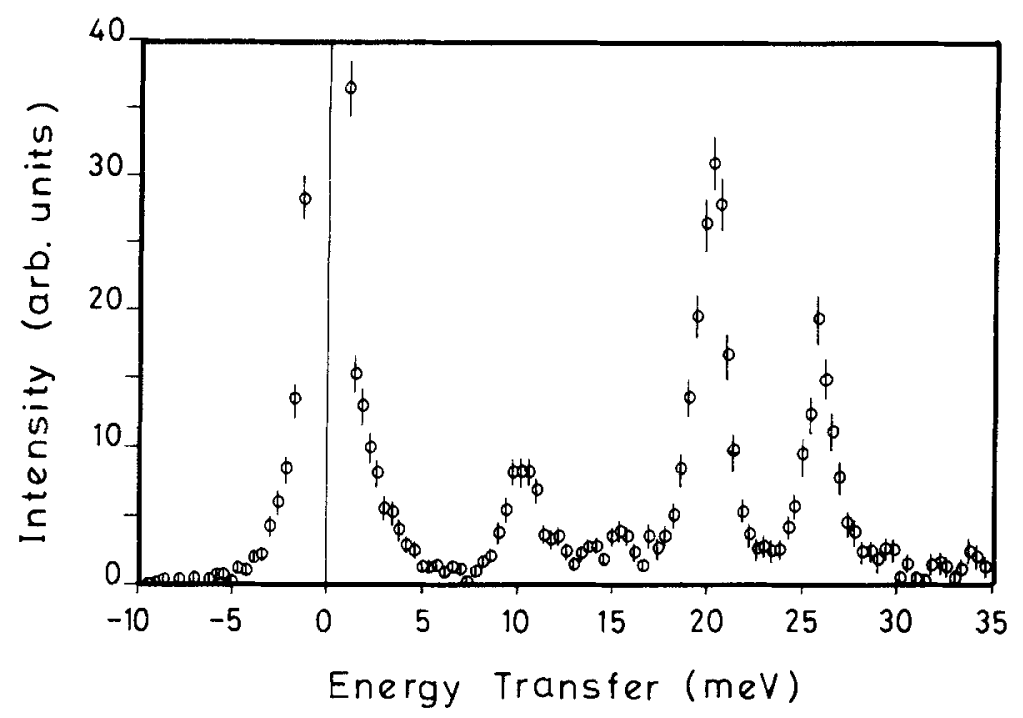

Figure 2. Crystal field transitions of $\mathrm{Nd}_{1.85} \mathrm{Ce}_{0.15} \mathrm{CuO}_{4}$ at $-1.9 \mathrm{~K}$.

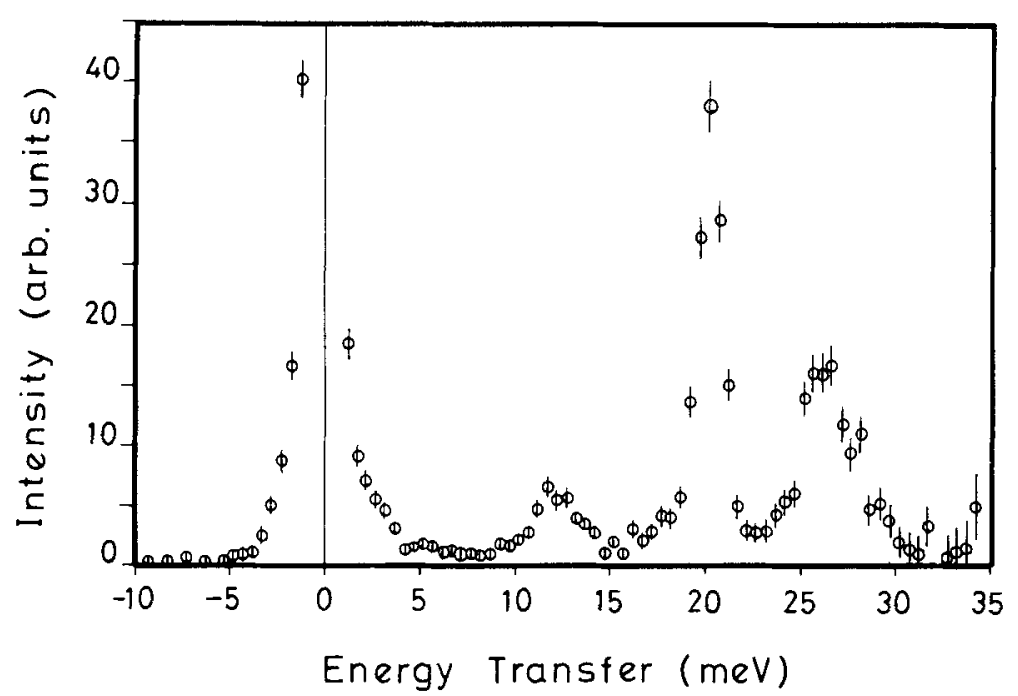

Figure 3. Crystal field transitions of $\mathrm{Nd}_{2} \mathrm{CuO}_{3.7} \mathrm{~F}_{0.3}$ at $1.9 \mathrm{~K}$.

21,27 and $93 \mathrm{meV}$, but in the doped samples an extra peak is seen at 10 to $12 \mathrm{meV}$. These excitations were identified as magnetic in origin from the decrease in their intensity with increasing $Q$. The obvious similarities between the ground state excitations of the two compounds suggest that in $\mathrm{Nd}_{2} \mathrm{CuO}_{4}$ there exists a CEF level below $21 \mathrm{meV}$ but with a very small transition probability from the ground state. Indeed, such a level may be inferred from the presence of a peak at about $11 \mathrm{meV}$ in the spectrum of $\mathrm{Nd}_{2} \mathrm{CuO}_{4}$ (not shown) measured at $200 \mathrm{~K}$. This extra peak at high temperatures arises from transitions between the thermaily-populated first-excited level and one of either the 21 or $27 \mathrm{meV}$ levels. 
The starting parameters of the fit were the set of B-coefficients calculated from a point-charge model with the known geometrical coordination of the $\mathrm{Nd}^{3+}$ ion in the $\mathrm{Nd}_{2} \mathrm{CuO}_{4}$ structure; the CEF parameters were then allowed to vary independently in order to achieve the best fit. In this way sets of parameters were found which agreed well with the neutron spectra of $\mathrm{Nd}_{1.85} \mathrm{Ce}_{0.15} \mathrm{CuO}_{4}$ and $\mathrm{Nd}_{2} \mathrm{CuO}_{3.7} \mathrm{~F}_{0.3}$. A set of parameters was found for $\mathrm{Nd}_{2} \mathrm{CuO}_{4}$, though having poorer agreement with the measured intensities, that corresponded to a scheme with the first excited CEF level at $16 \mathrm{meV}$.

Whilst the above analysis in terms of the CEF Hamiltonian (1) represented a reasonable description of the data, some disturbing discrepancies between the calculated and measured spectra remained, especially for the $\mathrm{Nd}_{2} \mathrm{CuO}_{4}$ sample. In particular, the calculated values of peak intensities did not match the measured intensities. The crystal field Hamiltonian (1), only includes interactions between the rare-earth magnetic moment and the crystal electric field. Additional perturbations on the energy levels may be caused by interactions with magnetic fields, which in $\mathrm{Nd}_{2} \mathrm{CuO}_{4}$ can arise from magnetic ordering of the $\mathrm{Nd}$ and $\mathrm{Cu}$ moments. Muon spin rotation measurements (Luke et al 1989) show that the $\mathrm{Cu}$ moments order at about $300 \mathrm{~K}$ in $\mathrm{Nd}_{2} \mathrm{CuO}_{4}$, but that no static magnetic order exists above $4 \mathrm{~K}$ in the superconducting, Ce-doped compound. The nature of the ordering in $\mathrm{Nd}_{2} \mathrm{CuO}_{4}$ has been examined recently in neutron diffraction experiments from single crystals (Endoh et al 1989; Skanthakumar et al 1989) and from a powder (Rosseinsky et al 1989). The copper spins adopt a simple antiferromagnetic arrangement in the planes, but undergo reorientation transitions at about $80 \mathrm{~K}$ and $30 \mathrm{~K}$ in which the relative configurations of spins in adjacent layers change. Below $30 \mathrm{~K}$ the $\mathrm{Cu}$ spins return to the high-temperature structure and begin to couple to the $\mathrm{Nd}$ spins. Long-range order of the $\mathrm{Nd}$ spins sets in below $4 \mathrm{~K}$, with the same magnetic structure as the $\mathrm{Cu}$ spins.

The simplest approximation to account for the effects of magnetic ordering on the crystal field levels is to add to the Hamiltonian (1) a term that represents the energy of a Nd moment in a mean molecular field, $\mathbf{H}_{\mathrm{mf}}$. In the present analysis the spins are taken to lie parallel to [110], in which case the additional term may be simplified to

$$
H=H_{\mathrm{CEF}}-h_{\mathrm{mf}}\left(\hat{J}_{x}+\hat{J}_{y}\right) / \sqrt{ } 2,
$$

where $h_{\mathrm{mf}}$ is the molecular field parameter in energy units. A repeat of the fitting procedure with the mean field term gave significantly better agreement with the measured transitions than before, especially for $\mathrm{Nd}_{2} \mathrm{CuO}_{4}$, and enabled all the ground-state and excited-state transitions to be calculated correctly to within the uncertainty on the measurements. The calculated sets of CEF and molecular field

Table 1. Crystal field parameters (in meV).

\begin{tabular}{lccc}
\hline Parameter & $\mathrm{Nd}_{2} \mathrm{CuO}_{4}$ & $\mathrm{Nd}_{1 \cdot 85} \mathrm{Ce}_{0 \cdot 15} \mathrm{CuO}_{4}$ & $\mathrm{Nd}_{2} \mathrm{CuO}_{3 \cdot 7} \mathrm{~F}_{0 \cdot 3}$ \\
\hline$B_{2}^{0}$ & $0.912 \pm 0.006$ & $0.887 \pm 0.004$ & $0.960 \pm 0.03$ \\
$B_{4}^{0}$ & $(1.25 \pm 0.02) \times 10^{-2}$ & $(1.37 \pm 0.05) \times 10^{-2}$ & $(1.36 \pm 0.03) \times 10^{-2}$ \\
$B_{6}^{0}$ & $(2.09 \pm 0.04) \times 10^{-4}$ & $(1.91 \pm 0.01) \times 10^{-4}$ & $(1.06 \pm 0.06) \times 10^{-4}$ \\
$B_{4}^{4}$ & $(-2.82 \pm 0.04) \times 10^{-2}$ & $(-2.43 \pm 0.03) \times 10^{-2}$ & $(-3.71 \pm 0.05) \times 10^{-2}$ \\
$B_{6}^{4}$ & $(-2.77 \pm 0.05) \times 10^{-3}$ & $(-2.82 \pm 0.02) \times 10^{-3}$ & $(-2.76 \pm 0.06) \times 10^{-3}$ \\
$h_{\mathrm{mr}}$ & $0.31 \pm 0.04$ & $0.15 \pm 0.02$ & $0.86 \pm 0.07$ \\
\hline
\end{tabular}


parameters are listed in table 1 . In view of the alternative suggestions for the $\mathrm{Cu}$ spin arrangement, we also analysed the data with a molecular field parallel to [100], but found no significant differences from the results described below. From this result we conclude that the crystal field spectra are relatively insensitive to the field direction in the plane. A more detailed discussion of the fitting procedure can be found in Boothroyd et al (1989).

\section{Conclusions}

The main effect of applying a molecular field is to cause a small splitting of each of the five doublets. Such a splitting in a Kramer's ion cannot be produced by a lowering of the symmetry through a small structural distortion. The magnitude of this splitting varies from peak to peak, and whilst the two components of the peaks are not resolved in the neutron measurement it is perhaps significant that for $\mathrm{Nd}_{2} \mathrm{CuO}_{4}$ the splitting of the $27 \mathrm{meV}$ peak is predicted to be twice that of the $20 \mathrm{meV}$ peak, which is consistent with the observed linewidths of the two peaks.

One can only speculate as to the origin of the molecular field in the two compounds. At the lowest temperature where measurements were made it is possible that the $\mathrm{Nd}$ spins were ordered in the $\mathrm{Nd}_{2} \mathrm{CuO}_{4}$ sample, but this is unlikely with the superconducting samples. The non-zero values of $h_{\mathrm{mf}}$ for $\mathrm{Nd}_{1.85} \mathrm{Ce}_{0.15} \mathrm{CuO}_{4}$ and $\mathrm{Nd}_{2} \mathrm{CuO}_{3.7} \mathrm{~F}_{0.3}$ are rather puzzling, therefore, since there has been no evidence to date for static magnetic ordering of the $\mathrm{Cu}$ spins in the superconducting phase of a high-temperature superconductor. On the other hand, whilst the existence of a magnetic interaction at the Nd site requires an exchange coupling, it does not necessarily imply long-range magnetic order. It is possible that coupling between the $\mathrm{Nd}$ and $\mathrm{Cu}$ spins produces a form of short-range order, brought on by frustration effects, with an exchange energy much greater than the thermal energies available at low temperatures. Such order may persist over a timescale sufficiently long to cause a splitting of the crystal field levels. The observations of powder diffraction peaks persisting above the apparent ordering temperature of $\mathrm{Nd}_{2} \mathrm{CuO}_{4}$ may be evidence for this effect. It is tempting to identify this proposed short-range order with the two-dimensional magnetic fluctuations of the $\mathrm{Cu}$ moments included in several current theories of the mechanism of high temperature superconductivity.

\section{References}

Boothroyd A T, Doyle S M. Paul D McK, Misra D M and Osborn R 1990 Physica C165 17

Endoh Y, Matsuda M, Yamada K, Kakurai K, Hidaka Y, Shirane G and Birgeneau R J 1989 Phys. Rer. B40 7023

Furrer A. Brüesch P and Untemähner P 1988 Phys. Ret. B38 4616

Luke G M et al 1989 Nature (London) 33849

Rosseinsky M J, Prassides K and Day P $1989 \mathrm{~J}$. Chem. Soc. Chem. Commun. 1734

Sauz-Puche R, Norton M, White T R and Glaunsinger W S 1983 J. Solid State Chem. 50281

Seaman C L et al 1989 Physica C159 391

Skanthakumar S, Zhang H, Clinton T W, Li W-H, Lynn J W, Fisk Z and Cheong S-W 1989 Physica C160 124

Tokura Y, Takagi H and Uchida S 1989 Nature (London) 337345 